\title{
Masonic Cancer Center
}

National Cancer Institute

\section{Source}

National Cancer Institute. Masonic Cancer Center. NCI Thesaurus. Code C39552.

The Masonic Cancer Center combines population sciences, basic research, and translational cancer care with community outreach and educational activities. It also facilitates the research of its members, fosters multidisciplinary approaches to cancer, provides infrastructure support for cancer research, and develops new methods and strategies to reduce cancer incidence and mortality. The Center is part of the University of Minnesota's Academic Health Center and was designated as an $\mathrm{NCl}$ comprehensive cancer center in 1998. 